\title{
Activated TCRs remain marked for internalization after dissociation from pMHC
}

Daniel Coombs, Alexis M. Kalergis, Stanley G. Nathenson, Carla Wofsy and Byron Goldstein

Nature Immunology 3, 926-931 (2002).

The second sentence of the legend to Fig. 4 contained an error. This sentence should read: "Each point represents the indicated TCR-pMHC

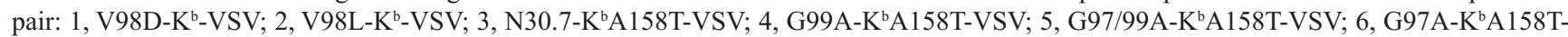

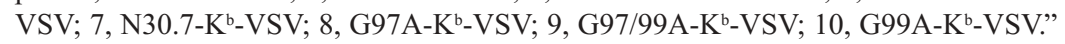

